\title{
Response: "Newborn chicks need no number tricks. Commentary: Number-space mapping in the newborn chick resembles humans' mental number line"
}

\author{
Rosa Rugani ${ }^{1,2 *}$, Giorgio Vallortigara ${ }^{1}$, Konstantinos Priftis $^{2}$ and Lucia Regolin ${ }^{2}$ \\ ${ }^{1}$ Center for Mind/Brain Sciences, University of Trento, Rovereto, Italy, ${ }^{2}$ Department of General Psychology, University of \\ Padova, Padova, Italy
}

Keywords: numerical cognition, number sense, domestic chick, spatial numerical associations, mental number line

\section{A commentary on}

Newborn chicks need no number tricks. Commentary: Number-space mapping in the newborn chick resembles humans' mental number line

by Shaki, S., and Fischer, M. H. (2015). Front. Hum. Neurosci. 9:451. doi: 10.3389/fnhum.2015.00451

OPEN ACCESS

Edited by:

Lorenzo Pia,

University of Turin, Italy

Reviewed by:

Barbara Treccani,

University of Sassari, Italy

*Correspondence:

Rosa Rugani

rosa.rugani@unitn.it;

rosa.rugani@unipd.it

Received: 13 October 2015 Accepted: 18 January 2016 Published: 05 February 2016

Citation:

Rugani R, Vallortigara G, Priftis K and Regolin L (2016) Response: "Newborn chicks need no number tricks. Commentary: Number-space

mapping in the newborn chick resembles humans' mental number line." Front. Hum. Neurosci. 10:31.

doi: 10.3389/fnhum.2016.00031
Newborn chicks do need number (magnitude) tricks, exactly like humans. Shaki and Fischer (2015) have argued that our results, according to which newborn chicks associate smaller numbers with the left space and larger numbers with the right space (Rugani et al., 2015), could be due to non-numerical cues such as area, perimeter, or density of the displayed dots. Regarding to our Experiment 3, they claimed "... chicks in all control conditions responded to both numerosity and at least one uncontrolled dimension, as they were in the first and second experiments, too." In Experiment 3 (Rugani et al., 2015) we actually performed three separate conditions to control for non-numerical cues. In the first condition, we controlled for shape, size, and color of each element. In the second condition we controlled for the overall area (summation of the areas of all elements depicted in each stimulus), and occupancy (the overall space occupied by each group of elements). In the third condition, we controlled for the overall perimeter (summation of the perimeters of all elements depicted in each stimulus), density (the mean distance among the elements), and occupancy. Notably, in the third condition a negative correlation was there between overall area and number: the overall area of the 32 elements was smaller than that of the 20 elements (training), and the overall area of the 20 elements was smaller than that of the 8 elements. Had chicks associated space to overall area, instead of number, their choices would have been the opposite to what we found. Thus, the association small numbers/left space and large numbers/right space holds even when the possible use of non-numerical cues is prevented, falsifying Shaki and Fischer's hypothesis.

Furthermore, chicks' behavior in the third condition explains why the supposed role of the light is unfounded. Shaki and Fischer (2015) stated that "There is an established association... of light with left... this "left-light bias" may explain why chicks turn to the left in the presence of fewer dots compared to their training." However, given that in one of our control condition there was a negative correlation between overall area and number (with perimeter and density held constant), smaller numbers were associated with larger (not smaller) areas and, therefore, with less light. Again chicks behaved the opposite to what predicted by Shaki and Fischer. Furthermore, the figure reported in Shaki and Fischer's comment is misleading. Indeed, this is a figure depicting the small versus large 
number test of our Experiment 1, and not one of the conditions, in which we controlled for the quantitative cues in Experiment 3.

Shaki and Fischer also suggested that the complexity, and not the number, of the patterns could explain the association with space: "less and more complex patterns are associated with left and right space (Beaumont, 1985; Heath et al., 2005)." We regard the comment as non-pertinent to our context, as these papers investigated aesthetic preferences in adult humans in front of stimuli depicting different figures. Beaumont (1985) asked participants to arrange two different pictures. Participants positioned the most complex figure on the left of the less complex one. We did not ask chicks to perform anything at all like that. Furthermore, the two stimuli employed in each test trial with chicks were identical: none could be considered neither more, nor less, "complex" than the other.

Heath et al. (2005) asked participants (Roman script readers [English], Arabic script readers, Biliterates, and Illiterates) to express their aesthetic preference by indicating one of two, vertically arranged, mirror compositions. Each composition was made by three different, horizontally arranged elements. Results showed different complex patterns of aesthetic preference, as a function of reading direction. We did not use this kind of stimuli with our chicks (i.e., on each trial we used two identical stimuli). Moreover, we did not ask our (illiterate) chicks to perform aesthetic judgments (i.e., we did not regard chicks' spontaneous choices as aesthetic judgments). Therefore, the "complexity" criticism by Shaki and Fischer is not founded.

Overall our results, in particular in the control conditions, are best explained by a bias that induces an association of smaller numbers with the left side and larger numbers with the right side. The main argument of Shaki and Fischer is that when dealing with non-symbolic numbers there are, unavoidably, continuous physical variables (i.e., sensory cues) that co-vary with number. This is certainly true. However, Approximate Number System (ANS) researchers do in fact think that numerosity estimates are

\section{REFERENCES}

Beaumont, J. G. (1985). Lateral organization and aesthetic preference: the importance of peripheral visual asymmetries. Neuropsychologia 23, 103-113. doi: 10.1016/0028-3932(85)90048-X

Gallistel, C. R. (2011). "Mental magnitudes," in Space, Time and Number in the Brain: Searching for the Foundations of Mathematical Thought, eds S. Dehaene and L. Brannon (New York, NY, Elsevier), 3-12.

Heath, R., Mahmasanni, O., Rouhana, A., and Nassif, N. (2005). Comparison of aesthetic preferences among Roman and Arabic script readers. Laterality 10, 399-411. doi: 10.1080/135765004420 00166

Rugani, R., Vallortigara, G., Priftis, K., and Regolin, L. (2015). Number-space mapping in the newborn chick resembles humans' mental number line. Science 347, 534-536. doi: 10.1126/science.aaa1379 computed from sensory cues. Indeed, different sensory properties combine to a common abstract (non-sensory) representation of number (or better, as we should point out below, of magnitudes). We believe that here resides our theoretical disagreement with Shaki and Fischer. They seem to believe that dimensions like duration, distance, location, brightness, and so on are not numbers. In contrast we believe, following Gallistel (2011), that they are indeed all numbers, in the sense that numbers are symbols for magnitudes. The SNARC effect is in fact so ubiquitous and multimodal, precisely because it deals with magnitude (quantity); magnitude includes numbers, but is not limited to it. Shaki and Fischer seem to attribute a particular status to numbers as representations of discrete quantity (numerosity). We believe, instead, that numbers are a part of a more general system for representing quantity, whether discrete or continuous. Gallistel (2011) has argued eloquently why it is necessarily to be so. Many continuous quantities are derived by an arithmetic combination of a discrete and a continuous quantity (e.g., rate). Therefore, the brain must possess a system to represent both discrete and continuous quantities within the same symbolic currency.

\section{AUTHOR CONTRIBUTIONS}

RR and GV wrote the manuscript. LR and KP provided critical revision. All authors approved the final version of the manuscript for submission.

\section{ACKNOWLEDGMENTS}

The original study was supported by the University of Padova (Progetto Giovani 2010, to RR, GRIC101142; and Progetto di Ateneo 2012 to R.L., CPDA127200) and by European Research Council (ERC) Advanced Grant PREMESOR ERC2011-ADG_20110406 to GV.

Shaki, S., and Fischer, M. H. (2015). Newborn chicks need no number tricks. Commentary: Number-space mapping in the newborn chick resembles humans' mental number line. Front. Hum. Neurosci. 9:451. doi: 10.3389/fnhum.2015.00451

Conflict of Interest Statement: The authors declare that the research was conducted in the absence of any commercial or financial relationships that could be construed as a potential conflict of interest.

Copyright (c) 2016 Rugani, Vallortigara, Priftis and Regolin. This is an open-access article distributed under the terms of the Creative Commons Attribution License (CC $B Y)$. The use, distribution or reproduction in other forums is permitted, provided the original author(s) or licensor are credited and that the original publication in this journal is cited, in accordance with accepted academic practice. No use, distribution or reproduction is permitted which does not comply with these terms. 